Published in final edited form as:

Gynecol Oncol. 2009 October ; 115(1): 135-137. doi:10.1016/j.ygyno.2009.06.012.

\title{
Tamoxifen and the Risk of Ovarian Cancer in BRCA1 Mutation Carriers
}

\author{
Danielle Vicus, Barry Rosen, Jan Lubinski, Susan Domchek, Noah D. Kauff, Henry T. \\ Lynch, Claudine Isaacs, Nadine Tung, Ping Sun, and Steven A. Narod Hereditary Ovarian \\ Cancer Clinical Study Group*
}

\begin{abstract}
Objective-BRCA1 mutation carriers have a high rate of both breast and ovarian cancer. Tamoxifen is a selective estrogen receptor modulator (SERM), which is used for the treatment of primary breast cancer and for the prevention of contralateral breast cancer. Our objective is to assess if tamoxifen treatment is associated with an increase in the subsequent risk of ovarian cancer among women with a BRCA1 mutation.
\end{abstract}

Methods-A matched case-control study was performed. Cases were 154 women with ovarian cancer and a previous history of breast cancer. Controls were 560 women with no ovarian cancer and a history of breast cancer. All cases and controls carry a deleterious BRCA1 mutation. Cases and controls were matched for year of birth, age at diagnosis of breast cancer and country of residence. The effect of tamoxifen treatment on the risk of subsequent ovarian cancer was estimated using conditional logistic regression.

Results-The unadjusted odds ratio for ovarian cancer, given previous tamoxifen treatment was 0.89 (95\% CI $0.54-1.49, p=0.66$ ). After adjusting for other treatments, the odds ratio was 0.78 (95\% CI 0.46-1.33, $p=0.36$ ).

Conclusion-Tamoxifen treatment for breast cancer does not appear to increase the risk of ovarian cancer in BRCA1 mutation carriers.

\section{Keywords}

Ovarian cancer; Tamoxifen; BRCA1 mutation

\section{Introduction}

Women who carry a BRCA1 mutation have an estimated 65-90\% risk of developing breast cancer and a 25-46\% risk of developing ovarian cancer [1], [2] and [3]. These rates are many times higher than the rates in the general population, and susceptible women are at risk for acquiring both types of cancer. After the diagnosis of breast cancer, we have estimated the 10-year actuarial risk of developing ovarian cancer in BRCA1 patients to be approximately $13 \%$ [4].

Corresponding author: Women's College Research Institute, 790 Bay Street, 7th Floor, Toronto, ON, Canada M5G 1N8. Fax: +1416 3513767.

Conflict of interest statement

Dr. Kauff reports receiving consulting fees and providing expert testimony for Wyeth. No other potential conflicts of interest are reported.

All other authors declare that there are no conflicts of interest. 
Tamoxifen is a selective estrogen receptor modulator (SERM), which is used for the prevention of primary, recurrent and contralateral breast cancer. Tamoxifen is, however, also associated with a two to three-fold increase in the risk of endometrial cancer in the general population [5] and [6] and in BRCA mutation carriers[7]. Through a feedback mechanism, tamoxifen causes prolonged periods of estrogenic stimulation, resulting in a state of hyperestrogenism. Additionally, it appears that tamoxifen exerts a direct agonist effect on estrogen receptors in the endometrium. Both of these effects likely contribute to the association between tamoxifen use and endometrial hyperplasia and cancer.

There is now evidence that estrogen replacement therapy is associated with an increased risk of ovarian cancer. In a large cohort study, Lacey et al. found a relative risk (RR) of 1.6 for ovarian cancer (95\% CI 1.2-2.0) among ever-users of estrogen replacement therapy and an RR of 3.2 (95\% CI 1.7-5.7) for those who had used estrogen replacement therapy for 20 years or more [8]. In the Million Women Study [9], current users of estrogen replacement therapy were significantly more likely to develop, or to die from ovarian cancer than were never-users (RR 1.34, 95\% CI 1.13-1.60 for incident disease and RR 1.48, 95\% CI 1.201.81 for death). However, in the National Surgical Adjuvant Breast and Bowel Project P-1 Study in which women with an increased risk of breast cancer were randomized to tamoxifen or placebo no difference in the incidence of ovarian cancer was found [6]. We believe that the effect of tamoxifen on the risk of ovarian cancer is still not clear and in need of further research. In particular, the issue is important for BRCA1 carriers, because many will be offered tamoxifen for treatment or for primary prevention and it is important to identify any potential risk factors that may contribute to the high risk of ovarian cancer. We asked whether or not treatment of breast cancer with tamoxifen was associated with an increase in the subsequent risk of ovarian cancer in women at inherited risk as the result of a BRCA1 mutation.

\section{Patients and Methods}

\section{Study population}

Eligible study participants were women who carry a deleterious mutation in the BRCA1 gene. Women were identified at the clinical genetics centers of affiliated hospitals in eight participating countries: Canada, France, Israel, Italy, Norway, Poland, UK and the USA. All women provided written informed consent. BRCA1 and BRCA2 mutation testing was performed in their country of residence. The participants completed a risk-factor questionnaire, which included information on their reproductive and medical histories. For women with a past history of breast cancer, this included type of surgery, chemotherapy, hormonal therapy, including tamoxifen and radiotherapy. Lifestyle factors including the use of oral contraceptives or hormone replacement therapy were also collected. The majority of participants completed the questionnaire during an outpatient visit to their genetics clinic. However, some completed it at home and mailed it to the study center. The ethics committees of all participating centers approved the study.

We restricted the study to 3038 women with a previous history of breast cancer (very few women without breast cancer had taken tamoxifen for chemoprevention). We excluded women who had ovarian cancer diagnosed before breast cancer $(n=59)$. We also excluded 421 subjects for whom data was missing on one or more key variables (tamoxifen use, year of breast or ovarian cancer diagnosis, oophorectomy or year of oophorectomy). The remaining 2558 women are the subjects of the present study. We did not include women with a BRCA2 mutation in this study due to the small number of women with ovarian cancer and a previous history of breast cancer. Among these breast cancer patients, cases were defined as women who had a subsequent diagnosis of ovarian cancer and controls were defined as women who did not later develop ovarian cancer. For each case, one or more 
control was selected; matched on date of birth ( \pm three years), age at diagnosis of breast cancer ( \pm three years) and country of residence. By this process, we generated 154 matched sets, comprised of 154 case patients with breast and ovarian cancer and 560 control patients with breast cancer only.

\section{Methods}

Cases and controls were compared for a number of variables, including date of birth, date of diagnosis of breast cancer, age at diagnosis of breast cancer, treatment for breast cancer (surgery, radiotherapy, chemotherapy) oral contraceptive use, hormone replacement treatment use and parity (Table 1). Student's $t$ test was used to test for statistical significance for continuous variables and the chi-square test was used for categorical variables. We calculated the odds ratio (OR) and 95\% confidence interval (CI) for ovarian cancer, given tamoxifen use, with unconditional logistic regression. A multivariable odds ratio was then estimated, adjusting for radiotherapy treatment (yes/no), chemotherapy (yes/no), type of breast cancer surgery (mastectomy vs. lumpectomy), age at diagnosis of breast cancer (trend), oral contraceptive use (yes/no), hormone replacement therapy use (yes/no) and parity $(0,1,2,3,4+)$. The multivariable adjusted odds ratios and $95 \% \mathrm{CI}$ were estimated using of SAS (version 9.1.3) and $p<0.05$ was considered to be statistically significant.

\section{Results}

Cases and controls are compared in Table 1. No differences were found in the average year of birth or age at diagnosis between the cases and controls. The distribution of types of treatments was similar for cases and controls. Approximately $20 \%$ of all the patients had been treated with tamoxifen. We performed univariable and multivariable analyses to assess the association between tamoxifen treatment and the risk of subsequent ovarian cancer. The odds ratio for ovarian cancer, given tamoxifen treatment was 0.89 (95\% CI $0.54-1.49, p=$ 0.66 ) in the univariable analysis (Table 2). In the multivariable analysis, we adjusted for radiotherapy, chemotherapy, breast cancer surgery (mastectomy vs. lumpectomy), age at diagnosis of breast cancer, oral contraceptive use, hormone replacement therapy use and parity. The odds ratio for ovarian cancer associated with tamoxifen treatment was 0.78 (95\% CI $0.46-1.33, p=0.36$ ).

\section{Discussion}

Tamoxifen has been shown to reduce the risk of distal recurrence in women with estrogenreceptor positive breast cancer by almost one-half, and to reduce the breast cancer mortality rate in these patients by one-third [10]. Tamoxifen has also been associated with a reduction in the risk of contralateral breast cancer in the general population and in BRCA1 carriers [11] and [12]. In our study, tamoxifen treatment for breast cancer was not associated with an increase in the subsequent risk of ovarian cancer. It is important to address this question because BRCA1 carriers are at increased risk for cancer at both sites. Metcalfe et al. found the 10-year actuarial risk of ovarian cancer after breast cancer in BRCA1 patients to be $12.7 \%$ [4].

The physiology of tamoxifen is complex; for example it has the properties of an estrogen antagonist in the breast epithelium, and as an agonist in the endometrium. This state of hyperestrogenism in the endometrium is known to cause hyperplasia and cancer. The physiological effect of tamoxifen on the ovary is less clear. Exogenous estrogen appears to increase the risk of ovarian cancer in post-menopausal women [8] and [9] and it is a concern that tamoxifen may exert a similar effect. Many ovarian cancers have been shown to be estrogen dependent with approximately 60\% expressing estrogen receptors [13] and [14]. In 
vitro, estrogen has been shown to mediate proliferation in human ovarian cell lines [15]. Tamoxifen is thought to have pro-estrogenic effects on the ovaries and currently is used to treat patients with recurrent, or persistent ovarian tumors. Hatch et al. treated 105 patients with advanced ovarian cancer with tamoxifen, whose disease had persisted or recurred after primary surgery and first-line chemotherapy [16]. They found a $10 \%$ complete response rate and an $8 \%$ partial response rate. Additional studies including previously treated patients with advanced ovarian cancer [17], [18] and [19] and asymptomatic patients with recurrent smallvolume cancer[20] found more modest response rates to tamoxifen treatment.

Cohen et al. reported an incidence of ovarian cancers of $5.7 \%$ (10/175) in post-menopausal breast cancer patients treated with tamoxifen, a rate 4-5 times the expected [21]. However, this was not confirmed in several additional studies, among them the National Surgical Adjuvant Breast and Bowel Project P-1 Study in which patients were randomized to tamoxifen or placebo [6], [22] and [23] All incident cancers were noted and no difference in the incidence of ovarian cancer was found. Our study is the first to evaluate the risk of ovarian cancer with tamoxifen treatment in BRCA1 mutation carriers. The strength of our paper is access to a unique population of women who are susceptible to both types of cancer. Currently, the majority of carriers of BRCA1 mutations undergo an oophorectomy to reduce their risk of ovarian cancer, but as many as 30-40\% of women with BRCA1 mutations do not elect to undergo the procedure [24]. Hence, it is important to study factors which potentially influence the risk of developing ovarian cancer. In conclusion, these data suggest that tamoxifen treatment can be offered to BRCA1 carriers for treatment or prophylaxis without increasing their risk of ovarian cancer.

\section{Acknowledgments}

Supported by a grant from the Canadian Breast Cancer Research Alliance, the Department of Defense Breast Cancer Research Program (DAMD17-03-1-0375 to N.D.K.), Project Hope for Ovarian Cancer Research and Education and The American Physicians Fellowship for Medicine in Israel.

\section{References}

1. Antoniou A, Pharoah PD, Narod S, Risch HA, Eyfjord JE, Hopper JL, et al. Average risks of breast and ovarian cancer associated with BRCA1 or BRCA2 mutations detected in case series unselected for family history: a combined analysis of 22 studies. Am J Hum Genet. 2003; 72:1117-1130. [PubMed: 12677558]

2. Risch HA, McLaughlin JR, Cole DE, Rosen B, Bradley L, Fan I, et al. Population BRCA1 and BRCA2 mutation frequencies and cancer penetrances: a kin-cohort study in Ontario, Canada. J Natl Cancer Inst. 2006; 98:1694-1706. [PubMed: 17148771]

3. King MC, Marks JH, Mandell JB. Breast and ovarian cancer risks due to inherited mutations in BRCA1 and BRCA2. Science. 2003; 302:643-646. [PubMed: 14576434]

4. Metcalfe KA, Lynch HT, Ghadirian P, Tung N, Olivotto IA, Foulkes WD, et al. The risk of ovarian cancer after breast cancer in BRCA1 and BRCA2 carriers. Gynecol Oncol. 2005; 96:222-226. [PubMed: 15589605]

5. Fisher B, Costantino JP, Redmond CK, Fisher ER, Wickerham DL, Cronin WM. Endometrial cancer in tamoxifen-treated breast cancer patients: findings from the National Surgical Adjuvant Breast and Bowel Project (NSABP) B-14. J Natl Cancer Inst. 1994; 86:527-537. [PubMed: 8133536]

6. Fisher B, Costantino JP, Wickerham DL, Redmond CK, Kavanah M, Cronin WM, et al. Tamoxifen for prevention of breast cancer: report of the National Surgical Adjuvant Breast and Bowel Project P-1 Study. J Natl Cancer Inst. 1998; 90:1371-1388. [PubMed: 9747868]

7. Beiner ME, Finch A, Rosen B, Lubinski J, Moller P, Ghadirian P, et al. The risk of endometrial cancer in women with BRCA1 and BRCA2 mutations. A prospective study. Gynecol Oncol. 2007; 104:7-10. [PubMed: 16962648] 
8. Lacey JV Jr, Mink PJ, Lubin JH, Sherman ME, Troisi R, Hartge P, et al. Menopausal hormone replacement therapy and risk of ovarian cancer. JAMA. 2002; 288:334-341. [PubMed: 12117398]

9. Beral V, Bull D, Green J, Reeves G. Ovarian cancer and hormone replacement therapy in the Million Women Study. Lancet. 2007; 369:1703-1710. [PubMed: 17512855]

10. Meyer UA, Zanger UM. Molecular mechanisms of genetic polymorphisms of drug metabolism. Annu Rev Pharmacol Toxicol. 1997; 37:269-296. [PubMed: 9131254]

11. Gronwald J, Tung N, Foulkes WD, Offit K, Gershoni R, Daly M, et al. Tamoxifen and contralateral breast cancer in BRCA1 and BRCA2 carriers: an update. Int J Cancer. 2006; 118:2281-2284. [PubMed: 16331614]

12. Metcalfe K, Lynch HT, Ghadirian P, Tung N, Olivotto I, Warner E, et al. Contralateral breast cancer in BRCA1 and BRCA2 mutation carriers. J Clin Oncol. 2004; 22:2328-2335. [PubMed: 15197194]

13. Clinton GM, Rougeot C, Derancourt J, Roger P, Defrenne A, Godyna S, et al. Estrogens increase the expression of fibulin-1, an extracellular matrix protein secreted by human ovarian cancer cells. Proc Natl Acad Sci U S A. 1996; 93:316-320. [PubMed: 8552629]

14. Rao BR, Slotman BJ. Endocrine factors in common epithelial ovarian cancer. Endocr Rev. 1991; 12:14-26. [PubMed: 1851084]

15. Baldwin WS, Curtis SW, Cauthen CA, Risinger JI, Korach KS, Barrett JCB. G-1 ovarian cell line: an alternative model for examining estrogen-dependent growth in vitro. In Vitro Cell Dev Biol Anim. 1998; 34:649-654. [PubMed: 9769151]

16. Hatch KD, Beecham JB, Blessing JA, Creasman WT. Responsiveness of patients with advanced ovarian carcinoma to tamoxifen. A Gynecologic Oncology Group study of second-line therapy in 105 patients. Cancer. 1991; 68:269-271. [PubMed: 2070324]

17. Weiner SA, Alberts DS, Surwit EA, Davis J, Grosso D. Tamoxifen therapy in recurrent epithelial ovarian carcinoma. Gynecol Oncol. 1987; 27:208-213. [PubMed: 3570058]

18. Markman M, Iseminger KA, Hatch KD, Creasman WT, Barnes W, Dubeshter B. Tamoxifen in platinum-refractory ovarian cancer: a Gynecologic Oncology Group Ancillary Report. Gynecol Oncol. 1996; 62:4-6. [PubMed: 8690289]

19. Karagol H, Saip P, Uygun K, Caloglu M, Eralp Y, Tas F, et al. The efficacy of tamoxifen in patients with advanced epithelial ovarian cancer. Med Oncol. 2007; 24:39-43. [PubMed: 17673810]

20. Markman M, Webster K, Zanotti K, Rohl J, Belinson J. Use of tamoxifen in asymptomatic patients with recurrent small-volume ovarian cancer. Gynecol Oncol. 2004; 93:390-393. [PubMed: 15099951]

21. Cohen I, Beyth Y, Tepper R, Shapira J, Zalel Y, Figer A, et al. Ovarian tumors in postmenopausal breast cancer patients treated with tamoxifen. Gynecol Oncol. 1996; 60:54-58. [PubMed: 8557228]

22. Cook LS, Weiss NS, Schwartz SM, White E, McKnight B, Moore DE, Daling JR. Populationbased study of tamoxifen therapy and subsequent ovarian, endometrial, and breast cancers. J Natl Cancer Inst. 1995; 87:1359-1364. [PubMed: 7658496]

23. McGonigle KF, Vasilev SA, Odom-Maryon T, Simpson JF. Ovarian histopathology in breast cancer patients receiving tamoxifen. Gynecol Oncol. 1999; 73:402-406. [PubMed: 10366467]

24. Metcalfe KA, Snyder C, Seidel J, Hanna D, Lynch HT, Narod S. The use of preventive measures among healthy women who carry a BRCA1 or BRCA2 mutation. Fam Cancer. 2005; 4:97-103. [PubMed: 15951959]

\section{Appendix: Other members of the Hereditary Ovarian Cancer Study Group}

M Daly (Fox Chase Cancer Center, Philadelphia, PA), J Garber (Dana-Farber Cancer Center, Boston, MA), C Kim-Sing (British Columbia Cancer Agency, Vancouver, BC), O Olopade (Department of Medicine, University of Chicago, Chicago, IL), S L Neuhausen (Epidemiology, Department of Medicine, University of California, Irvine, CA), W Foulkes (Program in Cancer Genetics, McGill University, Montréal, Quebec), P Ghadirian (Epidemiology Research Unit,(CHUM)- Hôtel-Dieu, Montréal, QC), D Gilchrist (University 
of Alberta, Edmonton, AB), S Merajver (University of Michigan Medical Center, Ann Arbor), S Manoukian (Istituto Nazionale Tumori, Milan), B Karlan (Cedars-Sinai Medical Center, Los Angeles, CA), J McLennan (University of California-San Francisco, CA), C Eng (Cleveland Clinic, Cleveland, OH), D Stoppa-Lyonnet (Institute Marie Curie, Paris), E Friedman (Oncogenetics Unit, Chaim Sheba Medical Center, Israel), A Eisen (Sunnybrook and Women's College Health Sciences Centre, Toronto, ON), G Rennert (Carmel Medical Center, Haifa, Israel), K Sweet (Clinical Cancer Genetics, Division of Human Genetics, General Internal Medicine, The Ohio State University, Columbus, OH), C Maugard (Hospital Notre Dame, Montréal, Quebec), F Couch (Mayo Clinic, Rochester, MN), R Gershoni-Baruch (Rambam Medical Center, Haifa), H Saal (Children's Hospital Medical Center, Cincinnati, Ohio), A Eisen (Toronto Sunnybrook Regional Cancer Centre, Toronto, Ontario), D. Rayson (Queen Elizabeth Health Sciences Centre, Halifax, Nova Scotia), P Ainsworth (University of Western Ontario/LRCC, London, ON), J Weitzel (City of Hope National Medical Center, Duarte, CA), D G Evans (St Mary's Hospital, Manchester), W Meschino (Preventive Oncology, Toronto-Sunnybrook Regional Cancer Centre, Toronto, ON), L Bordeleau (Department of Medical Oncology, University of Toronto, Toronto, ON), C Cybulski and J Gronwald (International Hereditary Cancer Center, Pomeranian Medical University, Szczecin, Poland, Dana Zakalik, Beaumont Research Institute, Detroit, MI. 
Table 1

Comparison of case and control subjects.

\begin{tabular}{llll}
\hline Variables & Controls $N=\mathbf{5 6 0}$ & Cases $N=\mathbf{1 5 4}$ & $\boldsymbol{P}$ value \\
Date of birth (range) & $1946.7(1916-68)$ & $1947.2(1919-66)$ & 0.62 \\
Diagnosis of breast cancer (range) & & \\
$\quad$ Year & $1989.2(1953-07)$ & $1989.1(1957-05)$ & 0.96 \\
Age & $43.0(26-68)$ & $42.5(27-66)$ & 0.53 \\
Chemotherapy & $61.6 \%$ & $66.8 \%$ & 0.29 \\
Radiotherapy & $46.5 \%$ & $50.7 \%$ & 0.42 \\
Type of surgery Mastectomy & $63.3 \%$ & $66.2 \%$ & 0.57 \\
Ovarian cancer diagnosis (range) & N/A & $1998.2(1978-08)$ & N/A \\
$\quad$ Year & N/A & $51.4(35-75)$ & N/A \\
Age & $20.7 \%$ & $20.1 \%$ & 0.89 \\
Tamoxifen treatment & $55.5 \%$ & $53.2 \%$ & 0.67 \\
Oral contraceptive use & $6.5 \%$ & $4.6 \%$ & 0.39 \\
Hormone replacement therapy & & \\
Parity & & $2.0(0-7)$ & 0.16 \\
Mean (range) & $2.2(0-8)$ & $13.6 \%$ & 0.50 \\
Nulliparity & $11.3 \%$ & & \\
\hline
\end{tabular}

All $p$ values were derived using the Student's $t$ test for continuous variables and the chi-square test for categorical variables. 
Table 2

Statistic analysis.

\begin{tabular}{lll}
\hline 154 BRCA1 mutation carriers matched sets & $\begin{array}{l}\text { Univariable analysis } \\
\text { OR }(\mathbf{9 5} \mathbf{C I}) \boldsymbol{p} \text { value }\end{array}$ & $\begin{array}{l}\text { Multivariable analysis } \boldsymbol{a} \\
\text { OR }(\mathbf{9 5} \mathbf{C I}) \boldsymbol{p} \text { value }\end{array}$ \\
Tamoxifen treatment (Y/N) & $0.89(0.54-1.49) 0.66$ & $0.78(0.46-1.33) 0.36$ \\
Chemotherapy (Y/N) & $1.39(0.89-2.17) 0.14$ & $1.23(0.76-2.00) 0.40$ \\
Radiotherapy (Y/N) & $1.29(0.86-1.92) 0.22$ & $1.32(0.80-2.15) 0.28$ \\
Type of surgery (mastectomy) & $1.17(0.72-1.90) 0.53$ & $1.23(0.68-2.23) 0.49$ \\
Age at diagnosis of breast cancer (trend) & $0.80(0.72-0.91) 0.0005$ & $0.82(0.72-0.93) 0.002$ \\
Oral contraceptive use (Y/N) & $0.85(0.51-1.40) 0.51$ & $0.84(0.49-1.44) 0.52$ \\
Hormone replacement therapy (Y/N) & $0.63(0.25-1.56) 0.32$ & $0.76(0.29-2.00) 0.57$ \\
Parity (trend) & $0.88(0.74-1.05) 0.13$ & $0.87(0.72-1.04) 0.11$ \\
\hline
\end{tabular}

${ }^{a}$ Multivariable analysis adjusted by oral contraceptive use (yes/no), hormone replacement treatment (yes/no), parity (trend), year of birth (trend), age at diagnosis of breast cancer (trend), radiotherapy (yes/no), chemotherapy (yes/no) and type of breast cancer surgery (mastectomy vs. lumpectomy). 